\title{
Hypoxic-State Estimation of Brain Cells by Using Wireless Near-Infrared Spectroscopy
}

\author{
Jinn-Rung Kuo, Bor-Shyh Lin, Member, IEEE, Chih-Lun Cheng, and Chung-Ching Chio
}

\begin{abstract}
Near-infrared spectroscopy (NIRS) is a modern measuring technology in neuroscience. It can be used to noninvasively measure the relative concentrations of oxyhemoglobin $(\mathrm{OxyHb})$ and deoxyhemoglobin (DeoHb), which can reflect information related to cerebral blood volume and cerebral oxygen saturation. Therefore, it has the potential for noninvasive monitoring of cerebral ischemia. However, there is still a lack of reliable physiological information on the relationship between the concentrations of $\mathrm{OxyHb}$ and DeoHb in cerebral blood and the exact hypoxic state of brain cells under cerebral ischemia. In this study, we describe a wireless multichannel NIRS system, which we designed to noninvasively monitor the relative concentrations of OxyHb and DeoHb in bilateral cerebral blood before, during, and after middle cerebral artery occlusion. By comparing the results with the lactate/pyruvate ratio measured by microdialysis, we investigated the correlation between the relative concentrations of $\mathrm{OxyHb}$ and DeoHb in cerebral blood and the hypoxic state of brain cells. The results showed that the relationship between the concentration changes of DeoHb in cerebral blood and the hypoxic state of brain cells was significant. Therefore, by monitoring the changes in concentrations of DeoHb, the wireless NIRS can be used to estimate the hypoxic state of brain cells indirectly.
\end{abstract}

Index Terms-Cerebra ischemia, hypoxic state, microdylasis, near-infrared spectroscopy (NIRS).

\section{INTRODUCTION}

$\mathbf{S}$ TROKE (brain attack) ranks as the third leading cause of disability and death worldwide. It can be classified into two major states: cerebral ischemia and hemorrhagic state [1], [2]. Previous studies have indicated that up to $80 \%$ of strokes result from cerebral ischemia due to occlusion of an artery (ischemia

Manuscript received October 25, 2012; revised December 31, 2012; accepted April 27, 2013. Date of publication May 2, 2013; date of current version December 31, 2013. This work was supported by the National Science Council of ROC under Contract 101-2221-E-009-039.

J.-R. Kuo is with the Division of Neurosurgery, Department of Surgery, Chi Mei Medical Center, Tainan 710, Taiwan, and also with the Department of Biotechnology, Southern Taiwan University, Tainan 710, Taiwan.

B.-S. Lin is with the Institute of Imaging and Biomedical Photonics, National Chiao Tung University, Tainan 711, Taiwan, and also with the Department of Medical Research, Chi Mei Medical Center, Tainan 710, Taiwan (e-mail: borshyhlin@gmail.com).

C.-L. Cheng is with the Institute of Imaging and Biomedical Photonics, National Chiao Tung University, Tainan 711, Taiwan.

C.-C. Chio is with the Division of Neurosurgery, Chi Mei Medical Center, Tainan 710, Taiwan, and also with the Department of Surgery, National Taiwan University Hospital, Taipei City 100, Taiwan.

Color versions of one or more of the figures in this paper are available online at http://ieeexplore.ieee.org.

Digital Object Identifier 10.1109/JBHI.2013.2261310 stroke), typically by blood clots in the middle cerebral artery (MCA) [3]. Cerebral ischemia results in low oxygen and glucose supply due to the decrease of blood flow, and it also causes a decrease in the production of adenosine triphosphate (ATP) due to a decrease of the acid cycle [4]-[6]. A lack of oxygen and glucose supply to the brain may lead to failure of energydependent processes and cause irreversible brain cell injury and death [7], [8]. Therefore, in order to prevent or diagnose ischemic strokes, it is important to monitor and understand the pathogenesis of cerebral ischemia.

In order to monitor ischemic strokes, several physiological information retrieval techniques have been developed [9], [10]. For example, microdialysis is a useful neuromedical technique used to monitor brain ischemia, hyperemia, hemorrhage, and even various kinds of physiological parameters in the brain. Microdialysis is a minimally invasive technique for sampling the chemistry of the interstitial fluid of organs and tissues. In 1990, Hillered et al. [11] first used microdialysis to monitor the chemistry in the brain tissue during surgical operations for tumors. By measuring glucose and glycerol metabolites, changes in the lactate/pyruvate ratio ( $L / P$ ratio) can provide physiological information related to the lack of oxygen in brain cells. Pyruvate in the cell is converted to acetyl-CoA via a pyruvate dehydrogenase reaction under aerobic condition or lactate under anaerobic condition. Therefore, the $L / P$ ratio has become an important parameter in diagnose of ischemic strokes. Although microdialysis can be applied to monitor ischemic strokes effectively, it also has the main disadvantages of being invasive and only being able to provide nonreal-time measurements.

Several noninvasive techniques such as magnetic resonance imaging (MRI), X-ray computed tomography (CT), and positron emission tomography (PET) have also been widely used to monitor variations in cerebral blood flow and oxygen metabolism in brain ischemia [10], [12], [13]. However, CT uses ionizing radiation and PET requires radioactive tracers to produce the images. Even though MRI is safer than CT and PET, its temporal resolution is obviously poorer and it is a more expensive procedure [14]. Moreover, none of these techniques is suitable for long-term monitoring of cerebral ischemia. Recently, near-infrared spectroscopy (NIRS) has been applied in cerebral science and neuroscience [15]. For near-infrared light, oxyhemoglobin $(\mathrm{OxyHb})$ and deoxyhemoglobin $(\mathrm{DeoHb})$ exhibit the most significant absorbance in the blood. Since blood delivers oxygen to tissue by local conversion of $\mathrm{OxyHb}$ and $\mathrm{DeoHb}$, the relative concentrations of $\mathrm{OxyHb}$ and $\mathrm{DeoHb}$ can reflect information related to cerebral blood flow, cerebral blood volume (CBV), and cerebral oxygen saturation [16]. Compared to PET, CT, and MRI, NIRS has the advantages of biochemical 
specificity, low cost, high temporal resolution, and the potential to measure intracellular and intravascular events simultaneously.

In 1993, Hirtz [17] first hypothesized that NIRS could be used to monitor stroke patients. Subsequent studies then started to investigate the application of NIRS systems on focal cerebral ischemia in rats [18]-[22]. In 1997, Wolf et al. [18] used NIRS to identify peri-infarct depolarizations from the concentration changes of $\mathrm{OxyHb}, \mathrm{DeoHb}$, and the oxidized form of cytochrome aa3. Vernieri et al. [19] performed NIRS and transcranial Doppler simultaneously to evaluate the effects of hypercapnia by monitoring the change of backscattered light intensity related to blood volume. Culver et al. [20] used a diffuse optical tomography device to concurrently obtain coregistered images of relative $\mathrm{CBV}$, tissue-averaged hemoglobin oxygen saturation, and relative cerebral blood flow under rat MCA occlusion. Xia et al. [21] used NIRS to assess the concentration changes of $\mathrm{OxyHb}$ and total-hemoglobin concentration (HbT) during acute focal cerebral ischemia and reperfusion. In 2010, Li et al. [22] developed a noninvasive electrooptical method using the peak changes in the relative absorption of pulsed near-infrared light to quantify brain oxygenation during ischemia and hypoxia in anesthetized rabbits. Most previous studies have focused on changes of $\mathrm{CBV}$, hemoglobin oxygen saturation, and cerebral blood flow under cerebral ischemia. However, there is still a lack of data on the relationship between the concentrations of $\mathrm{OxyHb}$ and $\mathrm{DeoHb}$ in cerebral blood and the hypoxic state of brain cells under cerebral ischemia.

In this study, we describe a wireless multichannel NIRS system that we designed to noninvasively monitor the relative concentrations of $\mathrm{OxyHb}$ and DeoHb in bilateral cerebral blood in real time, before, during, and after MCA occlusion. By comparing the results with the $L / P$ ratio measured by microdialysis, we investigated the correlation between variations in the concentration of $\mathrm{OxyHb}$ and $\mathrm{DeoHb}$ in cerebral blood and the hypoxic state of brain cells. Rat brain slices were stained with triphenyltetrazolium chloride (TTC) to confirm the irreversibly infarcted regions of the brain caused by MCA occlusion. The total hemoglobin concentration (HbT) for cerebral ischemia was investigated. This paper is organized as follows. The theoretical models of tissue optics and the implementation of the proposed wireless multichannel NIRS system are introduced in Section II. In Section III, the experiment design for the focal cerebral ischemia rat model is introduced. In Section IV, the correlations between $\mathrm{DeoHb}, \mathrm{OxyHb}$, and $L / P$ ratio before, during, and after MCA occlusion are investigated, and the total hemoglobin concentration for cerebral ischemia is also investigated. Finally, the conclusion is drawn in Section V.

\section{METHODS}

\section{A. Implementation of the Wireless Multichannel NIRS System}

We designed a wireless multichannel NIRS system to continuously monitor concentration variations of DeoHb and $\mathrm{OxyHb}$ under MCA occlusion, the system architecture of which is shown in Fig. 1, consisting of a light emitting-detecting probe

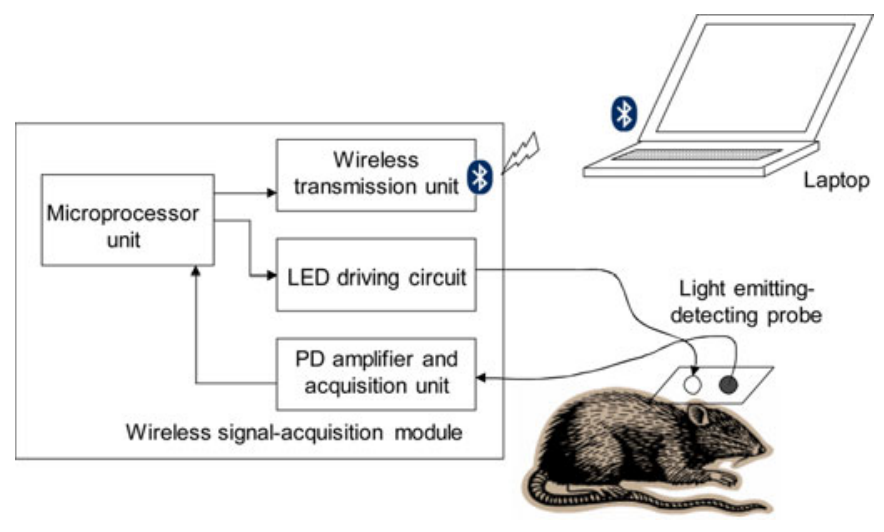

Fig. 1. System architecture of the proposed wireless multichannel NIRS system.

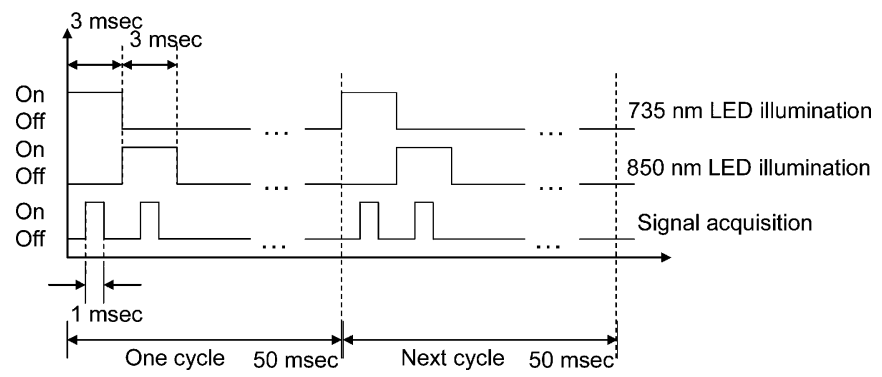

Fig. 2. Time-series diagram of dual-wavelength illumination and acquisition.

and a wireless signal acquisition module. The light emittingdetecting probe was designed to make contact with subjects or animals, provide a near-infrared light source, and detect diffusely reflected near-infrared light. The backboard of light emitting-detecting probe was made of rubber to provide high flexibility to adapt the head shape of the animal. And a disposable twin-adhesive plate is used to firmly bind the rat head and the contact side of the light emitting-detecting probe. The wireless signal acquisition module was designed to drive the nearinfrared light-emitting diode (SMT735/850, EPITEX, Japan), and amplify and acquire the near-infrared signal detected by the photodiode (PD15-22 C/TR8, EVERLIGHT, Taiwan). And each light source can provide about $0.3 \mathrm{~mW}$. The sampling rate is set to $20 \mathrm{~Hz}$, and the time-series diagram of dual-wavelength illumination and acquisition is shown in Fig. 2. The light emittingdetecting probe can be firmly attached to subjects or animals, and then the near-infrared light will penetrate through biological tissues where the light will be scattered and absorbed, and some pass through the tissues. The photodiode on the light emittingdetecting probe receives the diffusely reflected light on the same side of the light source, and this diffusely reflected light contains physiological information related to the biological tissues through which it penetrates. In general, the penetration depth is about half of the distance between the light source and detector. Therefore, the target location of where to monitor the concentration changes of OxyHb and DeoHb can be set accordingly [23]. Our multichannel NIRS system acquires and amplifies the intensity of diffusely reflected light, converts it from an analog signal to a digital signal and then transmits the signal wirelessly to a 


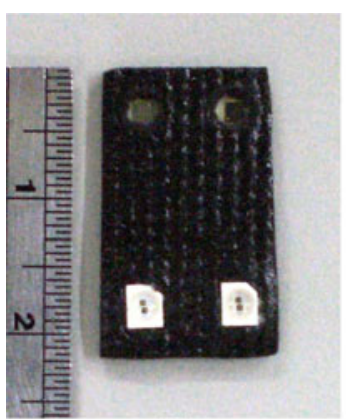

(a)

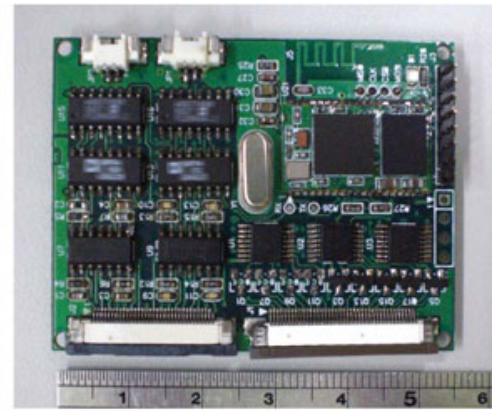

(b)
Fig. 3. (a) Light emitting-detecting probe and (b) wireless signal acquisition module.

computer or handheld device via Bluetooth. Finally, the changes in $\mathrm{OxyHb}$ and DeoHb concentrations are calculated using the modified Beer-Lambert law (MBLL). Photographs of the light emitting-detecting probe and wireless signal acquisition module are shown in Fig. 3(a) and (b), respectively.

\section{B. Theoretical Models of Tissue Optics}

The variations in the concentrations of $\mathrm{OxyHb}$ and DeoHb can be quantified by using the modified Beer-Lambert law. Previous studies have proven that the MBLL is an empirical description of optical attenuation in biological tissues. The variation of diffuse reflectance caused by variations in the concentrations of $\mathrm{OxyHb}$ or DeoHb [24], [25] can be expressed as

$$
\Delta \mathrm{OD}=-\log \frac{I_{f}}{I_{0}}=\varepsilon \Delta \mathrm{CLB}
$$

where $\Delta \mathrm{OD}$ is the variation of diffuse reflectance, and $I_{f}$ and $I_{0}$ are the final and initial intensities of diffusely reflected light, respectively. $\varepsilon$ and $\Delta C$ denote the extinction coefficient and the concentration change corresponding to specific chromophore, respectively. $L$ is the distance between emitting LED and PD, and $B$ is the differential path-length factor (DPF), which is a scaling factor relating to the light source-detector distance to the true optical path-length traveled by the scattered light. Absorption coefficient $\mu_{a}$ can be expressed as

$$
\mu_{a}=\sum_{i} \varepsilon_{i} C_{i}
$$

In the near-infrared wavelength range, the absorptions of water and lard are far less than those of OxyHb and DeoHb [26]. Therefore, the absorptions of OxyHb and $\mathrm{DeoHb}$ are mainly considered in the absorption model. Equation (1) then can be rewritten as follows:

$$
\Delta \mathrm{OD}^{\lambda}=\left(\varepsilon_{\mathrm{OxyHb}}^{\lambda} \Delta[\mathrm{OxyHb}]+\varepsilon_{\text {DeoHb }}^{\lambda} \Delta[\mathrm{DeoHb}]\right) B^{\lambda} L .
$$

Here, $\lambda$ denotes the wavelength of the emitted light, and $\Delta[\mathrm{OxyHb}]$ and $\Delta[\mathrm{DeoHb}]$ are the concentration changes of $\mathrm{OxyHb}$ and $\mathrm{DeoHb}$, respectively. In order to calculate $\Delta[\mathrm{OxyHb}]$ and $\Delta[\mathrm{DeoHb}]$, at least two different wavelengths are required, which can be calculated by

$$
\begin{aligned}
& \left.\Delta[\mathrm{OxyHb}]=\frac{\varepsilon_{\text {DeoHb }}^{\lambda_{1}}\left(\Delta \mathrm{OD}^{\lambda_{2}} / B^{\lambda_{2}}\right)-\varepsilon_{\text {DeoHb }}^{\lambda_{2}}\left(\Delta \mathrm{OD}^{\lambda_{1}} / B^{\lambda_{1}}\right)}{\left(\varepsilon_{\text {DeoHb }}^{\lambda_{1}} \varepsilon_{\text {Oxy }}^{\lambda_{2}}-\varepsilon_{\text {DeoHb }}^{\lambda_{2}} \varepsilon_{\text {Oxy }}^{\lambda_{1}}\right) L}\right) \\
& \Delta[\mathrm{DeoHb}]=\frac{\varepsilon_{\mathrm{OxyHb}}^{\lambda_{2}}\left(\Delta \mathrm{OD}^{\lambda_{1}} / B^{\lambda_{1}}\right)-\varepsilon_{\text {Oxy Hb }}^{\lambda_{1}}\left(\Delta \mathrm{OD}^{\lambda_{2}} / B^{\lambda_{2}}\right)}{\left(\varepsilon_{\text {DeoHb }}^{\lambda_{1}} \varepsilon_{\text {Oxy }}^{\lambda_{2}}-\varepsilon_{\text {DeoHb }}^{\lambda_{2}} \varepsilon_{\text {Oxy }}^{\lambda_{1}}\right) L} .
\end{aligned}
$$

The wavelengths of $\lambda_{1}$ and $\lambda_{2}$ have to straddle the isosbestic point, which is the superposition of the absorption spectra of $\mathrm{OxyHb}$ and $\mathrm{DeoHb}$, and they were set as 735 and $850 \mathrm{~nm}$, respectively, in this study. Under the assumption that all biological tissues are a semi-infinite medium [25], the parameter $B$ can then be expressed by,

$$
B=\frac{1}{2}\left(\frac{3 \mu_{s}^{\prime}}{\mu_{a}^{\text {initial }}}\right)^{1 / 2}\left[1-1 /\left(1+L\left(3 \mu_{s}^{\text {initial }} \mu_{a}^{\text {initial }}\right)^{1 / 2}\right]\right.
$$

where $\mu_{s}^{\prime}$ is the transport scattering coefficient. Equation (6) shows that $B$ depends on the tissue scattering coefficient and initial chromophore concentration. The parameter $B$ can be regarded as being independent to $\mu_{a}$ due to that the parameter $B$ is empirically determined and the influence of $\mu_{a}$ is actually small [25]. Finally, the change $\Delta[\mathrm{HbT}]$ in $\mathrm{HbT}$ can be obtained by

$$
\Delta[\mathrm{HbT}]=\Delta[\mathrm{OxyHb}]+\Delta[\mathrm{DeoHb}] .
$$

\section{EXPERIMENTS FOR FOCAL CEREBRAL ISCHEMIA RAT MODELS}

\section{A. Animals Preparation}

Adult male Sprague-Dawley rats (weight: $325 \pm 25 \mathrm{~g}$ ) were used in this experiment. All of the rats were kept under a 12$\mathrm{h}$ light/12-h dark cycle, and allowed access to food and water freely. All experimental procedures conformed to the guidelines of the National Institute of Health, Taiwan, and were approved by the Animal Care and Use Committee of Chi Mei Medical Center to minimize discomfort to the rats during surgery and the recovery period. The rats were randomly assigned to two groups: Group $1(n=6)$ was monitored using the proposed NIRS system under MCA occlusion; and Group $2(n=6)$ was monitored using microdialysis. All of the animals were anesthetized with sodium pentothal ( $25 \mathrm{mg} / \mathrm{kg}$, intraperitoneal (i.p.); Sigma Chemical Co., St Louis, MO) and a mixture containing ketamine (44 mg/kg, intramuscular (i.m.); Nan Kuang Pharmaceutical, Tainan, Taiwan), atropine $(0.02633 \mathrm{mg} / \mathrm{kg}$, i.m.; Sintong Chemical Industrial Co. Ltd., Taoyuan, Taiwan), and xylazine (6.77 mg/kg, i.m.; Bayer, Leverkusen, Germany). All of the rats were sacrificed on the third day postsurgery, and rat brain slices were stained with TTC solution.

\section{B. Experiment Design for Ischemic Stroke}

In order to research human stroke, several models of focal cerebral ischemia have been developed, and it has been found 


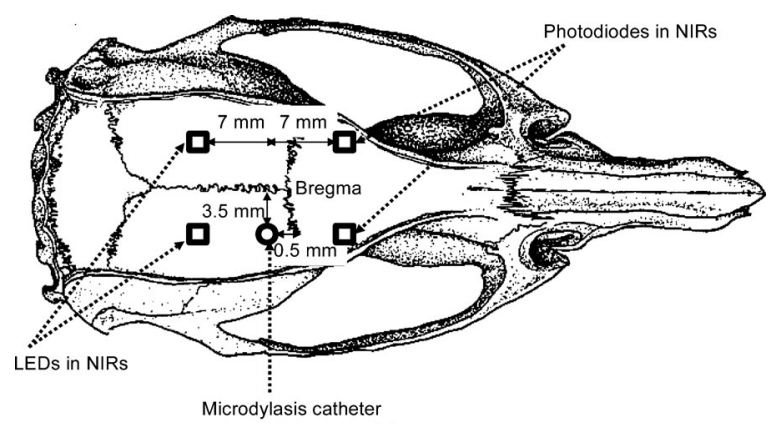

(a)

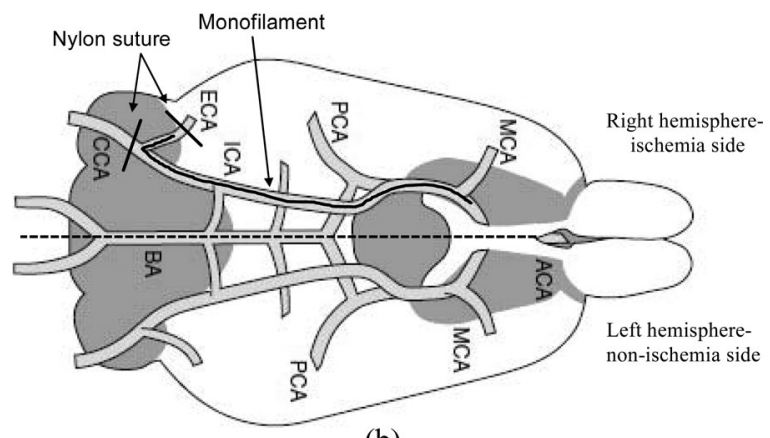

(b)

Fig. 4. (a) Monitoring positions were at the striatum region of rat brains, anterior-posterior $-0.5 \mathrm{~mm}$ and lateral $+3.5 \mathrm{~mm}$ from the bregma, under the top of the rat brain by $7 \mathrm{~mm}$ (includes $1 \mathrm{~mm}$ rat skull). Microdialysis catheter inserted under the top of the rat brain by approximately $5-6 \mathrm{~mm}$. The distance between the LEDs and PDs was set to $14 \mathrm{~mm}$. (b) Illustration of bilateral CCA occlusion. Sketch modified from current protocols in [3]. Anterior cerebral artery (ACA), basilar artery (BA), CCA, ECA, ICA, MCA, and posterior cerebral artery (PCA).

that up to $80 \%$ of strokes result from ischemic damage in the MCA area [3]. Therefore, MCA occlusion has become the main prototype in focal cerebral ischemic rat models. In this study, we also used MCA occlusion as the focal cerebral ischemic rat model. Before MCA occlusion, the rats were first anesthetized, and then the head was placed in a stereotaxic frame, and inserted and tightened by the ear bars. Next, a rectal temperature probe attached to the thermostatic controller was inserted into the colon of the rat to maintain the core temperature at $37{ }^{\circ} \mathrm{C}$. The locations that the light emitting-detecting probe and the microdialysis catheter were placed are shown in Fig. 4(a).

The experimental procedure of cerebral ischemia followed the experimental design of Belayev et al. [27]. For MCA occlusion, the rat was reversed, and a 25-mm surgical midline incision was made to expose the right common carotid artery (CCA), external carotid artery (ECA), and internal carotid artery (ICA), and a 4-0 nylon suture was used to dissect the artery from surrounding nerves and fascia under an operating microscope. Two knots of 4-0 nylon suture were tied around the right CCA and ECA to obstruct blood flow, and then a small puncture was opened in the right ECA, and a $23 \mathrm{~mm}$ monofilament suture was inserted from the right ECA through the ICA until the tip occluded the MCA [see Fig. 4(b)]. Before MCA occlusion was performed, microdialysis and the proposed wireless NIRS system recorded 60 and $10 \mathrm{~min}$ of baseline data, respectively. MCA occlusion surgery was completed within 5-10 min, and

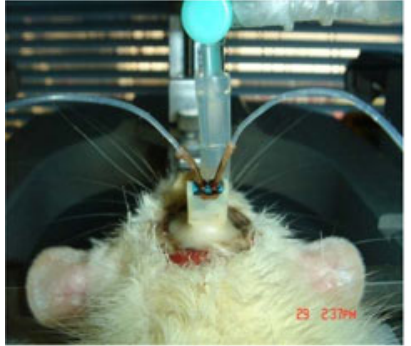

(a)

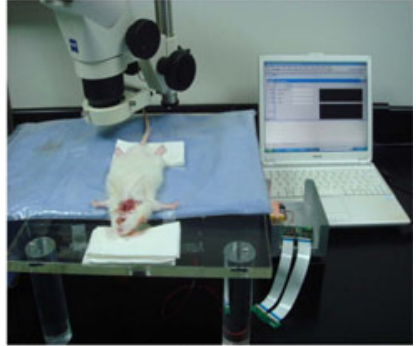

(b)
Fig. 5. Rat monitored by (a) microdialysis and (b) the proposed NIRS system under MCA occlusion.

the rats were then monitored continuously for 60 min under MCA occlusion. Next, we removed the monofilament suture to produce MCA reperfusion. The rats were still monitored continuously for $60 \mathrm{~min}$ after reperfusion. After all experimental procedures, all incisions on the rats were sutured with surgical wound sutures. Fig. 5(a) and (b) shows the photographs of a rat monitored by microdialysis and our NIRS system, respectively, under MCA occlusion.

\section{RESUltS AND DiscusSIONS}

The correlations between the changes of $\mathrm{OxyHb}$, DeoHb, and $L / P$ ratio under MCA occlusion and reperfusion were investigated to determine the exact hypoxic state of brain cells with regards to the concentration changes of $\mathrm{OxyHb}$ and $\mathrm{DeoHb}$ in cerebral blood. Fig. 6(a) and (b) shows the temporal profiles of $\Delta[\mathrm{OxyHb}]$ and $\Delta[\mathrm{DeoHb}]$ obtained from the ischemia side and contralateral side (nonischemia side), respectively, during the MCA occlusion. The results showed that $\Delta[\mathrm{OxyHb}]$ dropped and $\Delta[\mathrm{DeoHb}]$ increased immediately during MCA occlusion, indicating that the cerebral blood of the rats was under extremely low oxygen supply during this period. $\Delta[\mathrm{OxyHb}]$ and $\Delta[\mathrm{DeoHb}]$ still maintained a lower and a higher concentration than their baseline values, respectively, during MCA occlusion. During MCA reperfusion, $\Delta[\mathrm{OxyHb}]$ and $\Delta[\mathrm{DeoHb}]$ gradually increased and decreased, respectively, and the value of $\Delta[\mathrm{OxyHb}]$ became higher than that for $\Delta[\mathrm{DeoHb}]$. This indicates that the oxygenated blood was flowing into the brain through the MCA. However, $\Delta[\mathrm{OxyHb}]$ dropped at about $6 \mathrm{~min}$ after MCA reperfusion, and $\Delta[\mathrm{DeoHb}]$ increased at the same time. Subsequently, $\Delta[\mathrm{DeoHb}]$ returned to a higher value than $\Delta[\mathrm{OxyHb}]$. Compared to Fig. 6(b), the changes of $\Delta[\mathrm{OxyHb}]$ and $\Delta[\mathrm{DeoHb}]$ in the contralateral side were not significant during MCA occlusion and reperfusion.

Next, the time courses of average and standard errors of mean (SEM) of $\Delta[\mathrm{OxyHb}], \Delta[\mathrm{DeoHb}]$ and the total hemoglobin concentration $\Delta[\mathrm{HbT}]$ in the ischemia and contralateral sides were analyzed and are shown in Fig. 7(a) and (b), respectively. Repeated-measure analysis of variance (ANOVA) for differences between time points and groups was used to analyze experimental data, and significance was set at a value of $P$ $<0.05$. The results showed that the changes of $\Delta[\mathrm{OxyHb}]$ and $\Delta[\mathrm{DeoHb}]$ in the ischemia side were statistically significant. At the beginning of MCA occlusion, $\Delta[\mathrm{OxyHb}]$ in 


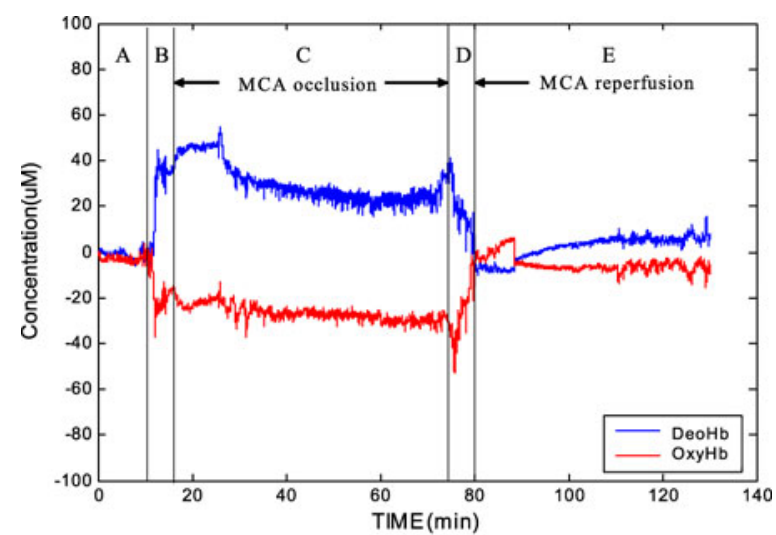

(a)

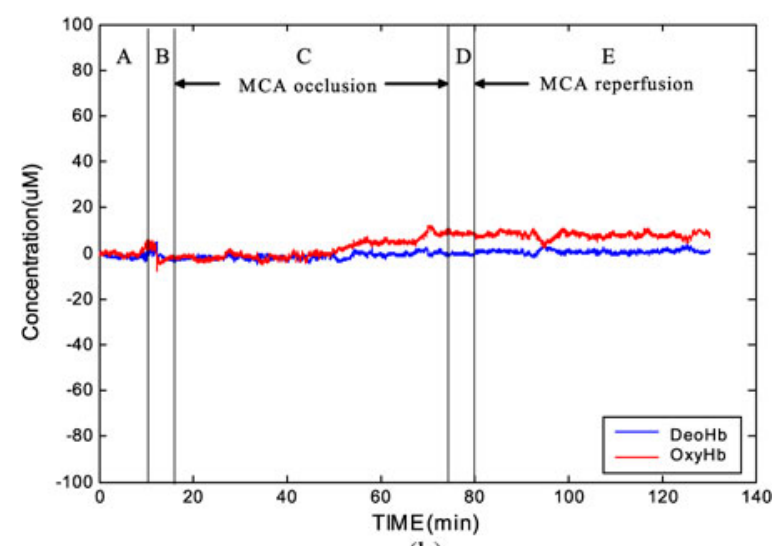

(b)

Fig. 6. Temporal profiles for concentration variation of $\Delta[\mathrm{OxyHb}]$ and $\Delta[\mathrm{DeoHb}]$ obtained from the (a) ischemia side and (b) nonischemia side under MCA occlusion. Period A: Before MCA occlusion (10 min), B: MCA occlusion surgery ( $5 \mathrm{~min}), \mathrm{C}$ : MCA occlusion (60 min), D: MCA reperfusion surgery (5 min), E: MCA reperfusion $(60 \mathrm{~min})$

the ischemia side dropped from $3.05 \pm 1.90$ to $-16.66 \pm$ $8.48 \mu \mathrm{M}$, and $\Delta[\mathrm{DeoHb}]$ increased from $2.55 \pm 1.67$ to 25.53 $\pm 8.97 \mu \mathrm{M} . \Delta[\mathrm{OxyHb}]$ increased to $9.75 \pm 10.17 \mu \mathrm{M}$ gradually, and $\Delta[\mathrm{DeoHb}]$ dropped to $3.4 \pm 5.46 \mu \mathrm{M}$ during MCA reperfusion. However, $\Delta[\mathrm{OxyHb}]$ then decreased gradually and became lower than $\Delta[\mathrm{DeoHb}]$ and its baseline within $10 \mathrm{~min}$. In the contralateral side, $\Delta[\mathrm{OxyHb}]$ was slightly lower than $\Delta[\mathrm{DeoHb}]$ during MCA occlusion, and then increased slightly during MCA occlusion [see Fig. 7(b)]. Finally, $\Delta[\mathrm{OxyHb}]$ remained slightly higher than $\Delta[\mathrm{DeoHb}]$ during the period of MCA reperfusion. The total hemoglobin concentration $\Delta[\mathrm{HbT}]$ is related to the relative total blood volume in living tissue [28]. The results showed that the variations of $\Delta[\mathrm{HbT}]$ in the ischemia side and contralateral side were slight during MCA occlusion and reperfusion.

The time course of the $L / P$ ratio $(n=6)$ monitored using microdialysis is shown in Fig. 8. The MCA occlusion control and sham data were recorded from the ischemia side and contralateral side, respectively. The results showed that the change of $L / P$ ratio was also statistically significant in the ischemia side. The $L / P$ ratio increased dramatically from $32.42 \pm 16.54$ to 323.48 \pm 118.08 during MCA occlusion, and the difference between MCA occlusion control and sham data was about 291.06. The

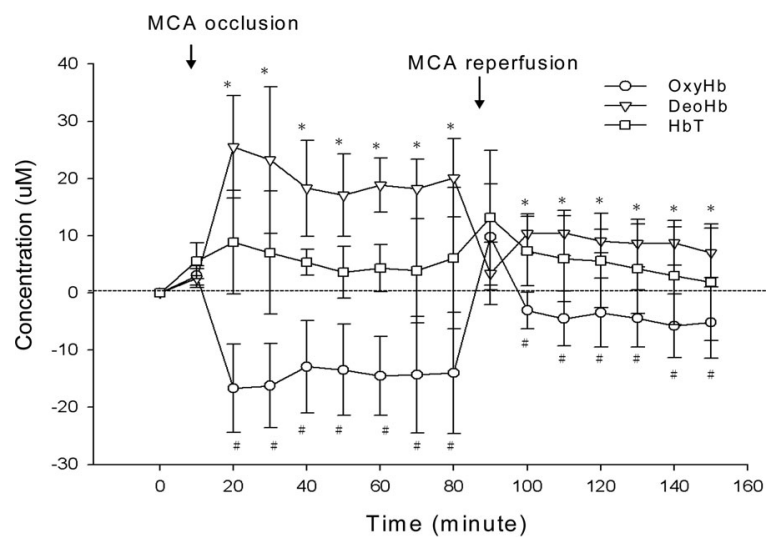

(a)

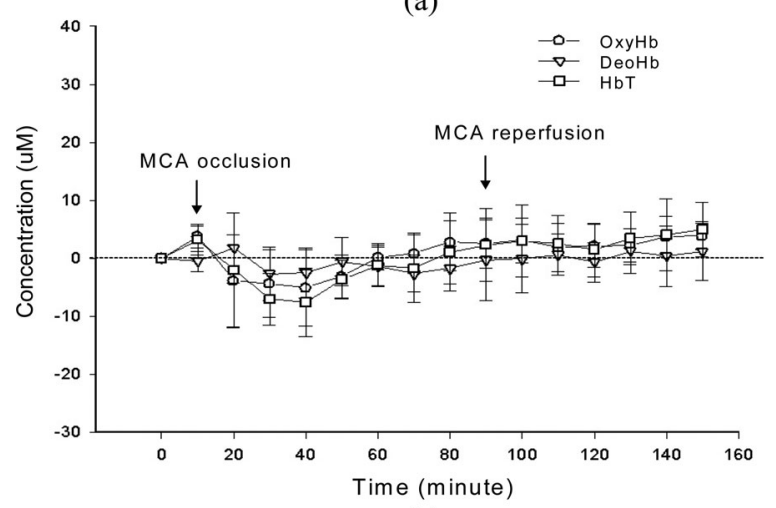

(b)

Fig. 7. Time courses for $\Delta[\mathrm{OxyHb}], \Delta[\mathrm{DeoHb}]$ and $\Delta[\mathrm{HbT}]$ in the (a) ischemia and (b) nonischemia sides ( $*$ and $\#$ mean $p<0.05$ for ANOVA).

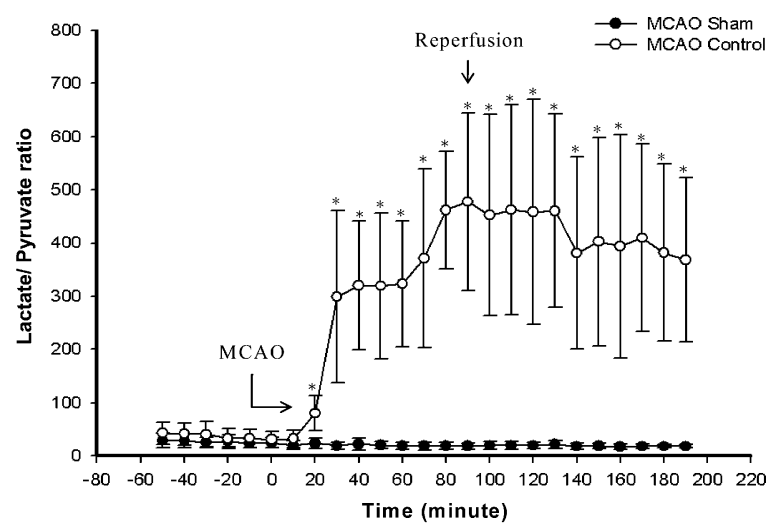

Fig. 8. Time courses for the $L / P$ ratio in ischemia (control) and nonischemia (sham) sides.

infarcted regions (ischemia side) and normal regions (contralateral side) in the rat brain slices with TTC staining are shown in Fig. 9, and the striatum regions of the brains have been marked. This clearly indicates that the low oxygen supply to the striatum region caused irreversible brain cell injury or death.

The correlations between $L / P$ ratio, $\Delta[\mathrm{OxyHb}]$ and $\Delta[\mathrm{DeoHb}]$ are shown in Fig. 10(a) and (b). The results showed that the correlation between $L / P$ ratio and $\Delta[\mathrm{OxyHb}]$ was $-0.60(p=0.0005)$, and the correlation between $L / P$ ratio and $\Delta[\mathrm{DeoHb}]$ was $0.82(p=0.0000)$. Although the time courses of $\Delta[\mathrm{OxyHb}]$ and $\Delta[\mathrm{DeoHb}]$ after MCA reperfusion were 


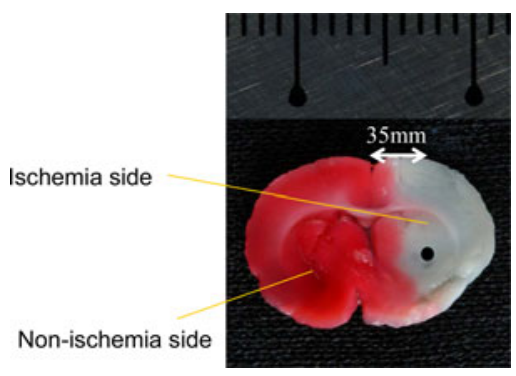

Fig. 9. TTC stain of the infarcted region (ischemia side) and normal region (nonischemia side). The circle marks the striatum region.

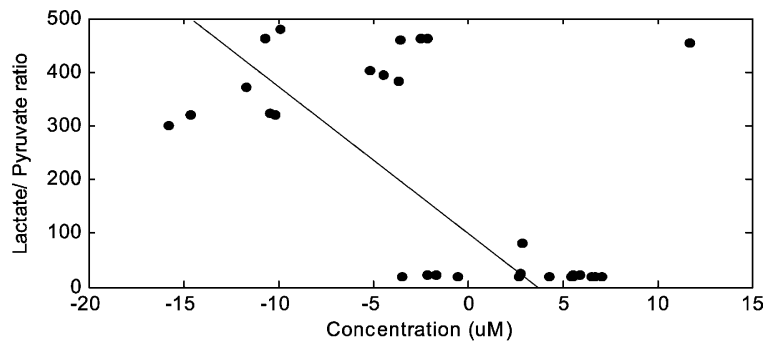

(a)

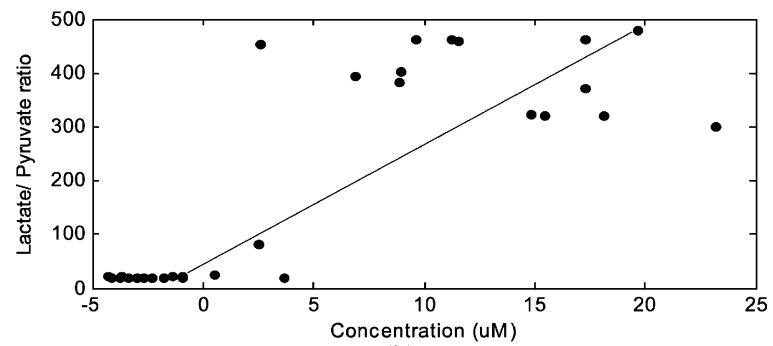

(b)

Fig. 10. (a) Correlation between $L / P$ ratio and $\Delta[\mathrm{OxyHb}]$, and (b) correlation between $L / P$ ratio and $\Delta[\mathrm{DeoHb}]$.

different from that of $L / P$ ratio, the correlation between $L / P$ ratio and $\Delta[\mathrm{DeoHb}]$ was high and the correlation between $L / P$ ratio, $\Delta[\mathrm{OxyHb}]$, and $\Delta[\mathrm{DeoHb}]$ was still significant.

The results of a study by Zaharchunk et al. [28] also indicated that the reduction of microvascular $\mathrm{CBV}$ is more significant than that of total CBV after MCA reperfusion. In edema, intravascular cell aggregates such as platelets and polymorphonuclear leukocytes, rheological changes due to hemoconcentration, endothelial microvilli formation, and vasomotion due to the release of vasoactive mediators have all been postulated to lead to a postischemic decrease in cerebral perfusion [29]. Therefore, during the main trunk of MCA occlusion period, cells distal to the occlusion area became ischemia, which would induce blood flow diverted from the microvasculature bed to thoroughfare channels, causing a decrease in capillary perfusion and a shift in blood volume from the microvasculature to this ischemia area. And it also results in the sight increase of HbT during MCA occlusion. The stability of $\Delta[\mathrm{HbT}]$ was maintained after MCA reperfusion.

Comparing the value of $\Delta[\mathrm{OxyHb}]$ and $L / R$ ratio during MCA reperfusion period, it showed that the $\Delta[\mathrm{OxyHb}]$ became higher; however, the $L / R$ ratio still remained high (477 \pm 166.76) during reperfusion period. This discrepancy may be due to multiple factors as followings. During the occlusion period, a reduction in the oxygen supply results in a severe failure of metabolic energy support, which leads to structure damage, cell ischemia, even cell death through reperfusion period [30]. Reperfusion and reoxygenation of the ischemia tissue may provide chemical substrate for further increasing cellular damage and even cell death [31]. Oxygen supplied by reperfusion was insufficient to meet the oxygen demand of the postischemia hypermetabolic state of cells after ischemia. [32], [33]. Therefore, the value of the $\Delta[\mathrm{OxyHb}]$ was higher but the $L / R$ ratio remained high $(477 \pm 166.76)$ during the reperfusion period.

\section{CONCLUSION}

In this study, we described a wireless multichannel NIRS system that we designed to noninvasively monitor the relative concentrations of OxyHb and $\mathrm{DeoHb}$ in bilateral cerebral blood before, during, and after MCA occlusion. By comparing the results with the $L / P$ ratio measured from microdialysis, the correlation between the relative concentrations of OxyHb and $\mathrm{DeoHb}$ in cerebral blood and the hypoxic state of brain cells were also investigated. The results showed that $\Delta[\mathrm{OxyHb}]$ decreased and $\Delta[\mathrm{DeoHb}]$ increased in the ischemia area during MCA occlusion. This indicates that the cerebral blood has an extremely low oxygen supply during MCA occlusion. The value of the $L / P$ ratio also increased dramatically during MCA occlusion, indicating the failure of metabolic reactions caused by the low oxygen and glucose supply to the rat brains. During the surgical period of MCA reperfusion, $\Delta[\mathrm{OxyHb}]$ and $\Delta[\mathrm{DeoHb}]$ in the ischemia area gradually increased and decreased, respectively, due to oxygenated blood flowing into the brain through the MCA. However, $\Delta[\mathrm{OxyHb}]$ dropped within a few minutes after MCA reperfusion, and became lower than $\Delta[\mathrm{DeoHb}]$ and its baseline. In contrast to the time courses of $\Delta[\mathrm{OxyHb}]$ and $\Delta[\mathrm{DeoHb}]$ after MCA reperfusion, the value of the $L / P$ ratio remained high. However, the correlation between $L / P$ ratio and $\Delta[\mathrm{DeoHb}]$ was still high. Therefore, by monitoring the changes in concentrations of DeoHb, the wireless NIRS can be used to estimate the hypoxic state of brain cells indirectly.

\section{REFERENCES}

[1] G. A. Donnan, M. Fisher, M. Macleod, and S. M. Davis, "Stroke," Lancet, vol. 371, pp. 1612-1623, 2008.

[2] N. R. Sims and H. Muyderman, "Mitochondria, oxidative metabolism and cell death in stroke," Biochim. Biophys. Acta, vol. 1802, pp. 80-91, 2010.

[3] M. J. O'Neill and J. A. Clemens, "Preclinical models of neurologic and psychiatric disorders-rodent models of focal cerebral ischemia," Curr. Protocols Neurosci., vol. 2000, pp. 9.6.1-9.6.32, 2000.

[4] M. E. Raichle, "The pathophysiology of brain ischemia," Neurol. Prog., vol. 13, pp. 2-10, 1983.

[5] J. Karkela, M. Pasanen, S. Kaukinen, P. Morsky, and A. Harmoinen, "Evaluation of hypoxic brain injury with spinal fluid enzymes, lactate and pyruvate," Critical Care Med., vol. 20, pp. 378-386, 1992.

[6] E. Ronne-Engstrom, H. Carlson, Y. S. Liu, U. Ungerstedt, and L. Hillered, "Influence of perfusate glucose concentration on dialysate lactate, pyruvate, aspartate, and glutamate levels under basal and hypoxic conditions: A microdialysis study in rat brain," J. Neurochem., vol. 65, pp. 257-262, 1995. 
[7] H. Ay, K. L. Furie, A. Singhal, W. S. Smith, A. G. Sorensen, and W. J. Koroshetz, "An evidence-based causative classification system for acute ischemic stroke," Ann. Neurol., vol. 58, pp. 688-697, 2005.

[8] P. Deba, S. Sharma, and K. M. Hassan, "Pathophysiologic mechanisms of acute ischemic stroke: An overview with emphasis on therapeutic significance beyond thrombolysis," Pathophysiology, vol. 17, pp. 197-218, 2010.

[9] J. A. Chalela, C. S. Kidwell, L. M. Nentwich, M. Luby, J. A. Butman, A. M. Demchuk, M. D. Hill, N. Patronas, L. Latour, and S. Warach, "Magnetic resonance imaging and computed tomography in emergency assessment of patients with suspected acute stroke: A prospective comparison," Lancet, vol. 369, pp. 293-298, 2007.

[10] C. J. Chen, F. C. Cheng, S. L. Liao, W. Y. Chen, N. N. Lin, and J. S. Kuo, "Effects of naloxone on lactate, pyruvate metabolism and antioxidant enzyme activity in rat cerebral ischemia/ reperfusion," Neurosci. Lett., vol. 287, pp. 113-116, 2000

[11] L. Hillered, L. Persson, U. Pontén, and U. Ungerstedt, "Neurometabolic monitoring of the ischaemic human brain using microdialysis," Acta Neurochirurgica, vol. 102, pp. 91-97, 1990.

[12] R. S. Frackowiak, G. L. Lenzi, T. Jones, and J. D. Heather, "Quantitative measurement of regional cerebral blood flow and oxygen metabolism in man using 150 and positron emission tomography: Theory, procedure, and normal value," J. Comput. Assisted Tomogr., vol. 4, pp. 727-736, 1980.

[13] J. A. Detre and D. C. Alsop, "Perfusion magnetic resonance imaging with continuous arterial spin labeling: Methods and clinical applications in the central nervous system,” Eur. J. Radiol., vol. 30, pp. 115-124, 1999.

[14] E. M. C. Hillman, "Optical brain imaging in vivo: Techniques and applications from animal to man," J. Biomed. Opt., vol. 12, pp. 051402-1051402-28, 2007.

[15] J. S. Wyatt, M. Cope, D. T. Delpy, C. E. Richardson, A. D. Edward, S. Wray, and E. O. Reynolds, "Quantitation of cerebral blood volume in human infants by near-infrared spectroscopy," J. Appl. Physiol., vol. 68, pp. 1086-1091, 1990

[16] C. W. Roy and C. S. Sherrington, "On the regulation of blood supply of the brain," J. Physiol., vol. 11, pp. 85-108, 1890.

[17] D. G. Hirtz, "Report of the national institute of neurological disorders and stroke workshop on near infrared spectroscopy," Pediatrics, vol. 91, pp. 414-417, 1993.

[18] T. Wolf, U. Lindauer, U. Reuter, T. Back, A. Villringer, K. Einhäupl, and U. Dirnagl, "Noninvasive near infrared spectroscopy monitoring of regional cerebral blood oxygenation changes during peri-infarct depolarizations in focal cerebral ischemia in the rat," J. Cereb. Blood Flow Metab., vol. 17, pp. 950-954, 1997.

[19] F. Vernieri, N. Rosato, F. Pauri, F. Tibuzzi, F. Passarelli, and P. M. Rossini, "Near infrared spectroscopy and transcranial doppler in monohemispheric stroke," Eur. Neurol., vol. 41, pp. 159-162, 1999.

[20] J. P. Culver, T. Durduran, D. Furuya, C. Cheung, J. H. Greenberg, and A. G. Yodh, "Diffuse optical tomography of cerebral blood flow, oxygenation, and metabolism in rat during focal ischemia," J. Cereb. Blood Flow Metab., vol. 23, pp. 911-924, 2003.

[21] M. Xia, S. Yang, J. W. Simpkins, and H. Liu, "Noninvasive monitoring of estrogen effects against ischemic stroke in rats by near-infrared spectroscopy," Appl. Opt., vol. 46, pp. 8315-8321, 2007.

[22] J. K. Li, T. Wang, and H. Zhang, "Rapid noninvasive continuous monitoring of oxygenation in cerebral ischemia and hypoxia," Cardiovasc. Eng., vol. 10 , pp. 213-217, 2010.

[23] F. Crespi, A. Bandera, M. Donini, C. Heidbreder, and L. Rovati, "Noninvasive in vivo infrared laser spectroscopy to analyse endogenous oxyhaemoglobin, deoxy-haemoglobin, and blood volume in the rat CNS," $J$. Neurosci. Methods, vol. 145, pp. 11-22, 2005.

[24] S. J. Matcher, M. Cope, and D. T. Delpy, "Use of the water absorption spectrum to quantify tissue chromophore concentration changes in nearinfrared spectroscopy," Phys. Med. Biol., vol. 38, pp. 177-196, 1993.

[25] D. A. Boas, T. Gaudette, G. Strangman, X. Cheng, J. J. A. Marota, and J. B. Mandeville, "The accuracy of near infrared spectroscopy and imaging during focal changes in cerebral hemodynamics," NeuroImage, vol. 13, pp. 76-90, 2001.

[26] R. Cubeddu, A. Pifferi, P. Taroni, A. Torricelli, and G. Valentini, "Noninvasive absorption and scattering spectroscopy of bulk diffusive media: An application to the optical characterization of human breast," Appl. Phys. Lett., vol. 74, pp. 874-876, 1999.

[27] L. Belayev, O. F. Alonso, R. Busto, W. Zhao, and M. D. Ginsberg, "Middle cerebral artery occlusion in the rat by intraluminal suture. Neurological and pathological evaluation of an improved model," Stroke, vol. 27, pp. 1616$1622,1996$.
[28] G. Zaharchunk, M. Yamada, M. Sasamata, B. G. Jenkins, M. A. Moskowitz, and B. R. Rosen, "Is all perfusion-weighted magnetic resonance imaging for stroke equal? The temporal evolution of multiple hemodynamic parameters after focal ischemia in rats correlated with evidence of infraction," J. Cereb. Blood Flow Metab., vol. 20, pp. 1341-1351, 2000.

[29] K. U. Frerichs, A. L. Siren, G. Z. Feuerstein, and J. M. Hallenbeck, "The onset of postischemic hypoperfusion in rats is precipitous and may be controlled by local neurons," Stroke, vol. 23, pp. 399-406, 1992.

[30] P. Lipton, "Ischemia cell death in brain neurons," Phys. Rev, vol. 79, pp. 1431-1568, 1999.

[31] I. Margaill, M. Plotkine, and D. Lerouet, "Antioxidant strategies in the treatment of stroke," Free Rad. Biol. Med., vol. 39, pp. 429-443, 2005.

[32] E. M. Nmato, K. A. Hossmann, and H. K. Cooper, "Post-ischemic hypermetabolism in cat brain," Stroke, vol. 12, pp. 666-676, 1981.

[33] W. Pulsinelli, "Pathophysiology of acute ischemic stroke," Lancet, vol. 339 , pp. 533-536, 1992

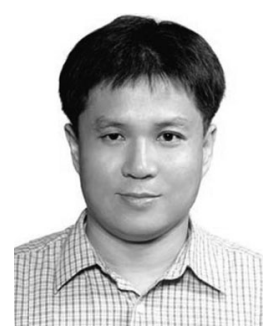

Jinn-Rung Kuo received the Medical degree from National Cheng Kung University, Tainan, Taiwan, in 1994, and the Ph.D. degree in medicine from the Graduate Institute of Clinical Medicine, National Cheng Kung University, in 2011.

$\mathrm{He}$ is currently an Associated Professor in the Department of Biotechnology, Southern Taiwan University, Tainan. His current research interests include neurotrauma and neurocritical care.

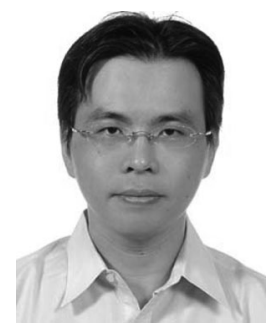

Bor-Shyh Lin (M'02) received the B.S. degree from National Chiao Tung University, Hsinchu City, Taiwan, in 1997, and the M.S. and Ph.D. degrees in electrical engineering from National Taiwan University, Taipei, Taiwan, in 1999 and 2006, respectively.

$\mathrm{He}$ is currently an Assistant Professor at the Institute of Imaging and Biomedical Photonics, Nationa Chiao Tung University. His current research interests include biomedical circuits and systems, biomedical signal processing, and biosensor

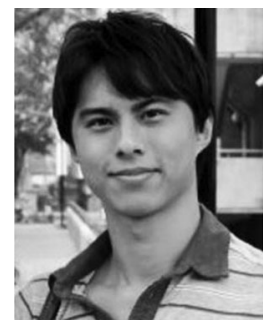

Chih-Lun Cheng received the B.S. degree from National Central University, Zhongli, Taiwan, in 2009 and the M.S. degree from National Chiao Tung University, Hsinchu City, Taiwan, in 2011.

He is currently with National Chiao Tung University. His current research interests include biomedical circuits and systems and biosensor.

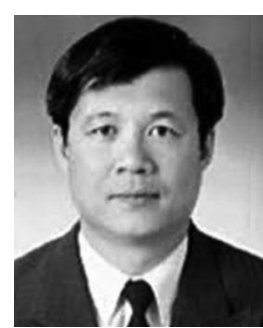

Chung-Ching Chio received the Medical degree from National Taiwan University, Taipei, Taiwan, in 1973.

He is currently a Professor in the Department of Surgery, National Taiwan University Hospital, Taipei, Taiwan. He is currently the Superintendent of Chi Mei Medical Center. His current research interests include neurotrauma and neurocritical care. 